\title{
Nefrologi, aggiornamento, EBM: analisi epistemologica e bioetica
}

\author{
Mario Timio ${ }^{1,2}$, Francesca Timio ${ }^{1}$ \\ ${ }^{1}$ Dipartimento di Medicina Interna e Nefrologia e Dialisi, Ospedale Foligno, Foligno (PG) \\ ${ }^{2}$ Direzione Scientifica Centro di Bioetica, Regione Umbria, Perugia
}

\begin{abstract}
Information and EBM in nephrology. Epistemology and bioethics
Medical knowledge is fluid and rapidly changing. The clinicians were under increasing pressure to keep up-todate and to base their practice more firmly on evidence, but few had the time necessary to do this. Then the problem of information is growing up more and more and recommendations are not appropriate in every situation. Differences in the clinical basis of individual patients and in local availability of intervention will always mean that the recommendation must be individually interpreted rather than applied systematically. However, the desire to be completely up-to-date needs to be balanced with the concern that a too rapid integration of the current studies may introduce bias, because studies with positive results are more likely to be published quickly. The philosophy of EBM system is based just on the scientific studies gathered in groups of clinical evidence to be applied to clinical practice. Evidence in nephrology is reported to be related to acute renal failure. Warnings are suggested to avoid to interpret EBM not as a medical gospel of XXI century, but only as a scientific up-to-date aid for clinical practice.
\end{abstract}

Keywords: Bioethics, EBM, Information, Nephrology

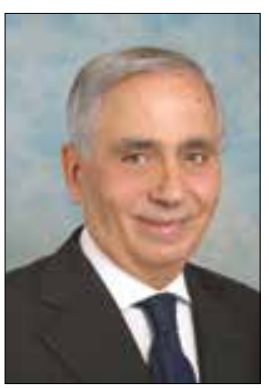

Esiste l'etica dell'aggiornamento in nefrologia?

Negli ultimi tempi c'è un po' di confusione nel campo della formazione medica continua (FMC). Da una parte, è forte la richiesta di trasparenza che arriva dai pazienti sull'obbligo formativo dei medici e, dall'altra, c'è la denuncia dei camici bianchi che accusano il "sistema" della loro scarsa adesione alla FMC a causa dell'insufficiente offerta e del loro poco tempo a disposizione per aggiornarsi a causa dei massacranti orari di lavoro. Sulla richiesta di trasparenza, i nefrologi hanno avuto uno stimolo continuo anche dall'Associazione degli Emodializzati (ANED), attenti alle esigenze dei nefropatici,

Accepted: June 30, 2016

Published online: July 25, 2016

Indirizzo per la corrispondenza:

Prof. Mario Timio

Via XX Settembre 22

06121 Perugia

timma@libero.it ma anche alle difficoltà dei medici di raggiungere il minimo previsto di aggiornamento. C'è addirittura chi vuole "sanzionare i medici non in regola e premiare chi matura più crediti con assunzioni e avanzamenti di carriera", determinando un serio punto di svolta per la Formazione Continua in Medicina. Di fatto, il ministro della salute, Beatrice Lorenzin, è orientato verso questa soluzione, alla quale è stata concessa una proroga addirittura dalla camera dei Deputati. Nel frattempo, è emersa la notizia che otre 6500 medici competenti sono stati depennati perché non in regola con i crediti formativi. Questi dati sono smentiti da Roberta Chersevani, Presidente FNOMCeO. Sembra proprio un caravanserraglio in cui tutti hanno ragione e nessuno recede dalla propria posizione. A complicare il quadro riemerge la problematica della Medicina Basata sulle Evidenze (EBM) con tutte le sue contraddizioni, innovazioni e criticità. Allora una domanda è d'obbligo. Dove si trovano la bioetica dell'aggiornamento del medico e la metodologia clinica? Dal primo punto, emerge la categoria della responsabilità. Essa coinvolge una serie di significati che è possibile far risalire alla sua stessa radice etimologica: responsabilità viene dal verbo latino rispondere (dare risposta), il quale rimanda anzitutto al soggetto chiamato direttamente in causa nell'esercizio della propria professione (rispondere in prima persona), ma reclama attenzione anche "all'altro", cioè al paziente (rispondere a qualcuno) e al contenuto dell'azione (rispondere di qualcosa: diagnosi, cura) (1). Queste tre 
componenti definiscono i criteri in base ai quali il giudizio bioetico deve essere formulato. Soggetto e oggetto, azione e relazionalità sono, infatti, alla radice dello statuto epistemologico della bioetica. Il che significa che solo dal ricorso ad essi (e non soltanto a uno di essi) è possibile pervenire a una seria valutazione bioetica della questione dell'aggiornamento (2). Perché aggiornarsi? Banale. Per il bene comune dei pazienti. II citato concetto di responsabilità richiama il primato della persona-malato e la necessità di restituire centralità alla coscienza e all'intenzionalità. L'esercizio delle responsabilità non può, però, avere come referente il solo criterio utilitaristico ( $a$ che cosa serve?), ma coinvolge più radicalmente la domanda circa il significato dell'atto (che senso ha?) e si inserisce, dunque, in un orizzonte molto più ampio della semplice fattività. Nell'etica dell'aggiornamento, c'è, quindi, l'etica della nuova civiltà medica e tecnologica. $\mathrm{E}$, nella nuova civiltà tecnologica, chi svetta al di sopra di ogni asticella culturale è l'architettura delle EBM.

\section{Approccio bayesiano alla medicina e tecnica dell'EBM}

L'approccio bayesiano alla medicina clinica è stato da anni rivisitato alla luce dell'EBM. Negli anni '90, parallelamente alla diffusione dell' $E B M$, si è assistito a una progressiva presa di coscienza della rilevanza clinica del ragionamento bayesiano e delle sue potenzialità e applicazioni. Se, poi, è riferito alla FMC, è stato rilevato che gli interventi EBM-dipendenti sono efficaci nel modificare la pratica clinica, diversamente dagli interventi puramente tradizionali. Con il passare del tempo, abbiamo, però, assistito a un ridimensionamento della pratica dell'EBM e dei suoi supporti tecnici e scientifici, come, per esempio, i grandi trial controllati, randomizzati e in doppio cieco (RCT) e successive metanalisi. E ciò essenzialmente perché, in molti trial, sono stati identificati dei bias che mettono in dubbio la congruenza clinica del trial stesso. Il tutto è esploso dopo la pubblicazione sul British Medical Journal di un articolo dal titolo molto intrigante: "Seeing what you want to see in randomized controlled trials" (3). Ma, allora, è proprio vero che ognuno può vedere, interpretare e trarre conclusioni cliniche a seconda di specifici punti di vista? Qual è la verità scientifica che un trial o RCT o metanalisi dovrebbero sottintendere? Sono illuminanti alcuni studi sull'insufficienza renale acuta (IRA) alla luce delle regole dell'EBM. Limitiamoci agli interventi terapeutici.

- Non ci sono RCT che valutino l'uso di fluidi nella prevenzione dell'IRA. Tuttavia, I'impiego di liquidi non è dannoso.

- Una revisione sistematica non ha trovato evidenze che I'uso di diuretici o dopamina riduca il rischio di IRA in pazienti con o senza sepsi.

- Due RCT hanno rilevato che l'impiego di diuretici peggiora il quadro clinico nella necrosi tubulare acuta e dopo interventi di cardiochirurgia.

- $\quad$ Non ci sono studi di evidenza che il mannitolo abbia effet-
tI sull'IRA in pazienti con rabdomiolisi traumatica o dopo un intervento di cardiochirurgia.

- Un vasto studio su pazienti con IRA trattati con peptidi natriuretici atriali non ha dimostrato vantaggi rispetto all'uso di placebo. Un altro trial ha mostrato un certo beneficio nei pazienti oligurici ma non in quelli non oligurici. Un altro studio ha smentito i benefici nei nefropatici con oliguria, tanto che è stato interrotto dopo un'analisi ad interim. Un RCT che testava l'impiego di Urodilantin, un peptide natriuretico con minori effetti emodinamici, non ha impedito l'accesso in dialisi rispetto a un gruppo trattato con placebo.

- L'acetilcisteina è inefficace nell'IRA da mezzi di contrasto; non esistono evidenze neanche in altre condizioni eziologiche.

- Ci sono scarse evidenze che l'emodialisi con membrane biocompatibili in pazienti con IRA riduca la mortalità più delle membrane non-biocompatibili. La dialisi continua contro quella intermittente non determina vantaggi (4).

\section{Quando la medicina basata sulle evidenze non funziona}

Di fronte a questa sequela di risultati EBM dipendenti talvolta contraddittori, il nefrologo come deve comportarsi? Rifarsi alle raccomandazioni basate sulle evidenze o fare ricorso al suo bagaglio culturale fatto di esperienze, che comprende errori e successi, vale a dire al ritorno della metodologia clinica? I medici sanno che i trial possono essere compromessi per I'introduzione di vari bias. Quello più comune nel processo di randomizzazione è la compromissione della fase di allocazione dei pazienti, che comporta almeno due problemi di ordine bioetico. II primo è insito proprio nella figura del paziente inserito nel protocollo di studio: egli diventa una figura sullo sfondo di una presunta scientificità, perdendone la centralità: sembra, anzi, che le decisioni relative alla persona oggetto di studio vengano prese da altri, riducendo la persona stessa a una delle variabili di un problema molto più complesso, quasi fossero, le persone, mere portatrici e produttrici di dati (5). $\mathrm{Se}$, poi, altre persone, e questo è il secondo impatto bioetico, debbano essere l'espressione clinica dei risultati del trial compromesso, è possibile che il trattamento sia inefficace se non dannoso. Si ritorna, allora, alla metodologia dei grandi clinici come Augusto Murri che, ai medici del suo tempo, scriveva: "C'è bisogno di pensare, riflettere e ragionare in ogni istante". Con questo, aveva in mente il metodo giusto per procedere e decidere sotto la luce di regole appropriate (6). Come si integrano le componenti suggerite da Murri con le regole dell'EBM? È sufficiente considerare la pratica dell'EBM come principio-guida al ragionamento clinico e all'approccio globale al paziente? Due pericoli si incontrano, oggi, nel giudizio dell'EBM e sono di segno opposto. Il primo è di evitare che I'EBM venga spacciata per un metodo diagnostico-terapeutico in grado di risolvere in termini epidemiologici e matema- 
tici i problemi fluttuanti dell'incerta pratica clinica. Non si può offrire ai medici un'immagine totalizzante dell'EBM e tantomeno un'idea di raccolta aggiornata delle attualità mediche o di panacea positivistica della medicina. È necessario far comprendere che l'EBM, pur essendo un procedimento standardizzato su basi scientifiche, è anche un sistema con molti limiti da tenere presenti nella pratica clinica. II pericolo di far considerare I'EBM come un totem o come un vangelo scientifico del XXI secolo o come distillato aggiornato delle attualità mediche $\mathrm{o}$, peggio, come il succedaneo spicciolo della metodologia clinica può essere evitato se si considera l'EBM solo come un completamento alla pratica clinica, rimovibile e sostituibile con altre tecniche che la scienza propone e che la storia della medicina puntualmente inserisce nel suo repertorio.

\section{Parole di The Lancet come conclusione}

Su The Lancet è riportato che "in alcune situazioni cliniche, la decisione giusta basata sulle evidenze induce a conseguenze che sono considerate immorali e contrarie agli interessi del paziente, perciò l'EBM non deve avere mai la precedenza su ragionate decisioni etiche prese dal medico a favore del paziente" (7). L'altro pericolo, di segno opposto, è che la pratica dell'EBM rappresenti solo un approfondimento culturale che scivola sulla realtà clinica, nel senso che non viene applicata nella pratica routinaria. II messaggio deve essere quello di calare l'EBM nella quotidianità clinica al fine di migliorare l'outcome di eventi patologici. Insegnare, praticare e incorporare nella pratica clinica l'EBM costituiscono, quindi, il miglior viatico per massimizzare la tecnica della Medicina Basata sulle Evidenze, senza dimenticare che le basali differenze biologiche, antropologiche, psicologiche e sociali nel singolo paziente suggeriscono che l'evidenza deve essere sempre interpretata individualmente piuttosto che essere applicata sistematicamente.

\section{Disclosures}

Financial support: No financial support was received for this submission.

Conflict of interest: The authors have no conflict of interest.

\section{Bibliografia}

1. Piano G. Per un nuovo modello etico. In AA.VV. Le Giornate di Bioetica (Di Pilla F., a cura di), Università di Perugia, Perugia 2010:175-85.

2. Ibidem.

3. McCormack J, Greenhalgh T. Seeing what you want to see in randomized controlled trials: version and perversion of UKPDS data. Brit Med J 2000;320:1720-3.

4. AA.VV. Clinical Evidence. Acute renal failure. BMJ Publishing Group. London 2001:634-48.

5. Gobbato CA. Verso un'Evidence-Based Medicine di supporto, ma non centrale. In AA.VV. Salute e Società, Franco Angeli. Milano 2006:206-9.

6. Murri A. Quattro lezioni e una perizia. II problema del metodo in medicina e biologia. Zanichelli, Bologna 1972:28.

7. Mant D. Evidence based medicine interventions and comprehensive treatment. Lancet 1998;353:743-6. 